\title{
TERAPIA OCUPACIONAL EN FRAGILIDAD: UNA APROXIMACIÓN BIBLIOGRÁFICA
}

\author{
Mirian Santamaría-Peláez \\ Universidad de Burgos \\ mspelaez@ubu.es \\ Jerónimo González-Bernal \\ Universidad de Burgos \\ jejavier@ubu.es
}

Recepción Artículo: 6 noviembre 2020 Admisión Evaluación: 10 noviembre 2020

Informe Evaluador 1: 25 noviembre 2020

Informe Evaluador 2: 23 noviembre 2020

Aprobación Publicación: 27 noviembre 2020

\section{RESUMEN}

El síndrome de fragilidad es una situación de alta prevalencia entre las personas mayores que afecta a todas las esferas de su funcionamiento; además es precursor de numerosos efectos adversos, incluso la dependencia o la muerte; pero, también se trata de una situación que puede ser revertida. La terapia ocupacional, como disciplina que persigue el funcionamiento óptimo de la persona en todas las actividades de su vida diaria, puede realizar intervenciones encaminadas a prevenir o revertir este estado de fragilidad. Se realiza una revisión con el objetivo de conocer la situación de la investigación en fragilidad desde el prisma de la terapia ocupacional. Tras una selección de artículos, se obtienen 9 que cumplen los criterios establecidos. Se concluye que la investigación en este campo es muy escasa aunque los resultados que ofrecen los estudios avalan la eficacia de las intervenciones de terapia ocupacional en personas mayores frágiles.

Palabras clave: fragilidad; terapia ocupacional; intervención; personas mayores; institucionalización.

\section{ABSTRACT}

Occupational therapy in frailty: a bibliographic approach. Frailty syndrome is a high prevalence situation among elderly people that affects performance; it is also precursor for adverse events, even dependence or death; but, it is also a situation that can be reversed. Occupational therapy, as a discipline that tends to an optimum performance in all activities of daily living, can carry out interventions focused in prevention or reversion of this frailty situation. With the aim to know the research situation about frailty from occupational therapy point of view, a revision is made. After a selection of the articles, 13 that comply established criteria are obtained. It is concluded that research in this field is very scarce although results obtained in studies endorse the effectiveness of occupational therapy interventions in frail elderly.

Keywords: frailty; occupational therapy; intervention; elderly people; institutionalization. 


\section{TERAPIA OCUPACIONAL EN FRAGILIDAD: UNA APROXIMACIÓN BIBLIOGRÁFICA}

\section{INTRODUCCIÓN}

Dentro del amplio rango de intervenciones profesionales que es posible realizar ante un caso de fragilidad (terapia física, farmacológica, nutricional, psicológica, etc.), se encuentra la terapia ocupacional (TO) como recurso para el tratamiento de la fragilidad como parte de una aproximación sistémica ante este síndrome.

La ocupación es todo lo que las personas hacen, las actividades que realizan en su día a día, bien para cuidar de sí mismos, contribuir al bienestar de su comunidad o cualquier otra que resulte importante 0 relevante para sus vidas (Fisher, Atler y Potts, 2007). Dentro del concepto de ocupación se encuentran enmarcadas las diferentes áreas de desempeño ocupacional, como son: actividades básicas de la vida diaria; actividades instrumentales de la vida diaria y educación, trabajo, ocio y participación social.

El rol del terapeuta ocupacional, al igual que en cualquier otra situación de salud, debe encontrarse enmarcado y respaldado por el trabajo en equipo de todos los profesionales que conforman el equipo multiprofesional. Por lo tanto, se tendrá en cuenta que los objetivos que se persiguen desde la intervención de la T0 deben ser consecuentes con los objetivos generales del plan de intervención y que este plan debe estar consensuado además de con el resto de profesionales, con la persona misma para conseguir que la intervención esté enmarcada dentro de un modelo de atención integral centrada en la persona.

Se debe tener en cuenta que la población con la que se trata se encuentra en una situación de vulnerabilidad debido tanto a su avanzada edad como a la situación de fragilidad; por tanto, se pretende poner énfasis en aqueIlos programas que aporten una mejoría significativa en lo referente a la calidad de vida de estas personas, siempre desde el punto de vista del desempeño ocupacional en ejecución de las diferentes actividades de la vida diaria.

\section{OBJETIVOS DE LA INVESTIGACIÓN}

El objetivo principal de esta investigación es conocer en qué punto se encuentra la investigación sobre fragilidad desde el punto de vista de la eficacia de las intervenciones de terapia ocupacional.

\section{MUESTRA}

Tras la búsqueda bibliográfica atendiendo a las palabras clave seleccionadas, se consideraron aptos para esta revisión un total de 9 artículos científicos publicados en diferentes revistas de divulgación científica y seleccionados según la metodología que se explica a continuación.

\section{METODOLOGíA}

La búsqueda de artículos incluidos en la revisión se realizó en dos bases de datos: EBSCO y NCBI (Pubmed). Para llevar a cabo la búsqueda se utilizaron las siguientes palabras en lengua española: síndrome de fragilidad, terapia ocupacional, fragilidad, ancianos frágiles, intervención terapia ocupacional en fragilidad; y las siguientes palabras en lengua inglesa: frailty, occupational therapy in frailty, elderly people, old people. Estos términos fueron combinados de diferentes maneras.

Los criterios de selección para la utilización de los artículos han sido principalmente que se tratara de artículos realizados desde la TO y con personas mayores en situación de fragilidad. No se plantea ningún criterio más debido a la escasez de documentación sobre el tema.

La búsqueda realizada arrojó un total de 25 artículos relevantes, sin contar los que aparecen repetidos en las diferentes combinaciones de búsqueda, de los cuales, tras aplicar los criterios anteriormente mencionados, solo se incluyeron 9. La principal razón de rechazo de artículos fue que no cumplían los criterios establecidos.

Entre los 13 artículos seleccionados aparecen principalmente artículos que versan sobre intervenciones desde la T0, aunque también se han incluido artículos de revisión en la bibliografía, pero principalmente debido a la escasez del material existente. Igualmente, no se ha descartado ningún artículo por la fecha de publicación. 


\section{RESULTADOS ALCANZADOS}

Fisher, Atler y Potts (2007) intentaron confirmar la efectividad de una intervención ocupacional a corto plazo. La intervención consistía en la realización de actividades diarias para mejorar indicadores asociados a la fragilidad. Sin embargo, los autores utilizan una definición más laxa (haber reportado necesitar ayuda para llevar a cabo actividades como: bañarse, prepararse la comida, trabajo pesado del hogar, compra de alimentos, transporte y manejo del dinero). Si bien no es la definición planteada fenotípicamente por Fried y cols. (1991; 2001), es utilizada también de manera válida para hacer referencia al estado de dependencia y vulnerabilidad.

En esta investigación participaron ocho adultos mayores de 65 años institucionalizados, que manifestaban indicadores relativos a la fragilidad y que no estaban recibiendo terapia ocupacional. La intervención consistió en cuatro sesiones de 45 minutos y se basó en el Modelo del Proceso de la Intervención de Terapia Ocupacional (OTIPM por sus siglas en inglés).

Para medir el desempeño en las actividades de la vida diaria (AVD), los investigadores utilizaron un instrumento observacional Ilamado AMPS (por sus siglas en inglés) que provee indicadores sobre habilidades motoras y habilidades de procesamiento requeridas para realizar una determinada tarea.

Los investigadores encontraron una diferencia estadísticamente significativa entre las medidas realizadas antes y después de la intervención ocupacional en cuanto a las habilidades motoras de las actividades diarias, pero no en cuanto a las habilidades de procesamiento de las AVD.

Se encontró en la investigación que aproximadamente en el 78 \% de todas las AVD evaluadas existía algún tipo de mejora, bien fuera porque las actividades habían vuelto a ser realizadas ( $22 \%$ de los casos) o porque, al menos, se había mejorado sustancialmente ( $55 \%$ de los casos). Es decir, la T0 redundó en una mejora sustancial en la realización de actividades ocupacionales de los adultos mayores.

En otra investigación llevada a cabo por Mann, Ottenbacher, Fraas, Tomita y Granger (1991), con una muestra de 104 ancianos se realizó una intervención a lo largo de 18 meses que se basó principalmente en la educación sobre la utilización de dispositivos asistenciales y modificaciones ambientales adaptadas a las necesidades del paciente.

En primer lugar, el terapeuta ocupacional visitaba la casa de la persona para realizar una evaluación ambiental; a partir de ahí ofrecía recomendaciones para la utilización de dispositivos o modificaciones del hogar, entrenamiento para su uso, y mantenía una comunicación continua de seguimiento a través de visitas cada seis meses en las que se evaluaban los resultados de la intervención.

Se pudo observar que existía una disminución en el declive funcional de los participantes, así como una disminución del costo de las hospitalizaciones e institucionalizaciones (porque estos presentaban problemas de menor gravedad). Además, se encontró que las personas objeto de la intervención mostraban una mayor independencia funcional en comparación con las que no la recibieron. También se halló que los no intervenidos tenían más gastos al ser ingresados en las instituciones y que empleaban más recursos económicos en la contratación de profesionales sociosanitarios.

De esta forma, se concluye que proveer a los adultos mayores de dispositivos tecnológicos de relativo bajo costo en conjunción con modificaciones ambientales en el hogar puede redundar en una mejora sustantiva en su calidad de vida, frenando el declive natural que es propio de la edad, especialmente al existir vulnerabilidad asociada producto de la fragilidad del anciano. La realización de actividades ocupacionales diarias ocurre entonces con mayor facilidad puesto que estos encuentran una efectiva asistencia no humana en sus procedimientos diarios, catalizando así su independencia y autoeficacia.

Más recientemente, Gitlin y cols. (2006), trabajando en una muestra de 319 adultos mayores frágiles, encontraron que una intervención enfocada en los factores de riesgo relacionados con las caídas y las discapacidades de los pacientes reducía las dificultades de la movilidad y las dificultades de AVD de los mismos. El programa de intervención consistía específicamente en entrenamiento de estrategias cognitivas, conservación de energía, agarre de barras de sujeción o barandillas, etc. 


\section{TERAPIA OCUPACIONAL EN FRAGILIDAD: UNA APROXIMACIÓN BIBLIOGRÁFICA}

De esta forma, se buscaba que los sujetos aumentaran su motivación para ejercer estrategias de afrontamiento para mantener o mejorar su desempeño en su vida diaria. El objetivo de la intervención tenía como meta la compensación en la realización de las actividades producto del declive a través de estrategias de control/mejoramiento que involucraban el aspecto cognitivo (resolución de problemas, reformulación), el comportamental (definición del propio ritmo, sentarse en vez de estar de pie al realizar una tarea), y el ambiental (inclusión de barras 0 barandas para que los adultos se apoyen).

Los terapeutas ocupacionales visitaban a los pacientes en sus hogares (cuatro veces en sesiones de 90 minutos) y, después de haber finalizado la intervención directa, establecían con ellos un contacto telefónico de alrededor de 20 minutos de duración para cerciorarse de que las pautas enseñadas seguían llevándose a cabo 0 para fortalecer las intervenciones acordadas.

A lo largo de las distintas sesiones, las intervenciones de los terapeutas ocupacionales consistían en entrenar directamente a la persona a través de las estrategias pautadas y monitorear y reforzar la ejecución de estas estrategias. En los últimos contactos (de intervención telefónica) se llevaba a cabo una evaluación sobre la solución de problemas, el uso refinado de las estrategias, y se facilitaban herramientas de educación y recursos para enfrentar situaciones adversas en el futuro.

Se implementaron además modificaciones ambientales como: instalación de barandillas, barras de apoyo para agarrarse en la casa, dispositivo elevador del asiento en el baño, etc. Aunque puede decirse que existían variaciones en la intervención debido a la individualidad de las necesidades de los pacientes, dicha intervención se sustentaba en cinco componentes principales: 1) educación y resolución de problemas, 2) modificaciones materiales del hogar, 3) técnicas de conservación de energía, 4) fortalecimiento de los músculos y del equilibrio y 5) técnicas de recuperación de caídas.

En conjunción con la T0, Ios pacientes eran también intervenidos con una terapia física (una sola visita de 90 minutos) que trataba sobre ejercicios de fortalecimiento de músculos y técnicas para recuperarse de las caídas.

Al hacer un análisis de los procedimientos seguidos en la intervención antes descrita, se visualizan perspectivas que deben guiar la TO en términos generales. Se observa que los adultos intervenidos eran ancianos que no recibían cuidado en su hogar y que eran catalogados como frágiles. Esto marca una diferencia con aquellos adultos mayores que pueden hacerse cargo de los costes que suponen cuidadores profesionales o que poseen familiares que cuiden de ellos o que se encuentran en instituciones que proveen este tipo de servicios.

Por otro lado, en la realización de la terapia existía una coordinación laboral entre los terapeutas ocupacionales y los fisioterapeutas. Al ser evaluada la efectividad del tratamiento, se observa en los resultados que estos pacientes (especialmente aquellos moderadamente vulnerables) redujeron su mortalidad en dos años en comparación con aquellos ancianos que no recibieron tal terapia.

El estudio anterior es relevante porque pone de manifiesto que cualquier intervención que se realice con los adultos mayores debe ser una acción multidisciplinaria donde los diversos profesionales abarquen problemas específicos de su área y en una tarea mancomunada puedan resolver efectivamente el problema.

Ledesma (2015) Ilevó a cabo una revisión sobre la T0 en el tratamiento de la sarcopenia para evaluar la efectividad de distintos procedimientos y cómo estos redundan en una mejoría de esta patología mencionada. La sarcopenia es un síndrome en el cual el adulto mayor manifiesta una pérdida progresiva de masa muscular con el consecuente declive en su fuerza física. Esto se vuelve un círculo vicioso en el anciano puesto que, al perder la fuerza física, el anciano pasa menos tiempo realizando actividades, y esta misma inactividad produce a su vez una pérdida mayor de masa muscular. Como los criterios fenotípicos expuestos por Fried y cols. (1991; 2001) tratan principalmente sobre la fragilidad física, una intervención encaminada a resolver este problema aborda también paralelamente el síndrome de la fragilidad en el anciano.

Ledesma (2015) hace referencia a varias intervenciones ocupacionales para el tratamiento de este síndrome. Entre las actividades por él investigadas se encuentran actividades en el jardín, en la cocina, bricolaje y taichi. 


\section{LA PSICOLOGÍA ANTE RETOS DE FUTURO. ABRIENDO CAMINOS}

Ledesma plantea que en la ejecución de estas actividades se desarrollan diversas destrezas como: destrezas sensorio-perceptuales; destrezas de regulación emocional; destrezas cognitivas; y destreza en comunicaciones sociales.

Ledesma (2015) explica que a través de un entrenamiento en taichi se mejora el equilibrio, se incrementa la fuerza, la coordinación, la flexibilidad, la postura y el funcionamiento físico de la persona mayor. Esto se consigue con los ejercicios habituales de esta práctica ya que se trabaja la musculatura de los miembros inferiores, se realizan posiciones con las rodillas flexionadas y hay cambios en los puntos de apoyo del peso del cuerpo. Este tipo de ejercicios ayudan a que los ancianos ejecuten con mayor calidad las AVD que supongan la bipedestación (y la bipedestación en sí misma), tales como ducharse, deambular o vestirse, mejorando de este modo su desempeño ocupacional en todas estas actividades.

En el caso del bricolaje, se trabaja la amplitud articular cuando se pintan grandes superficies, se trabaja la fuerza y la resistencia (si se trabaja con instrumentos como el martillo, el destornillador o la lija), pudiendo también desarrollarse la motricidad fina cuando se trabaja pintando detalles con un pincel fino. En la jardinería se trabaja la coordinación, la fuerza, el equilibrio y el rango de movimientos. Estos aspectos se trabajan con el cavado en la tierra, el podado, el trasplante de plantas, el vaciado y llenado de las macetas. Se trabaja el equilibrio rastrillando, la motricidad fina con la siembra de semillas o la eliminación de esquejes 0 hierbas indeseables, etc.

Las actividades de la cocina suponen un mejoramiento generalizado de la actividad física en el anciano porque, según Ledesma (2015), se trabaja el equilibrio, la motricidad fina, el rango articular, la fuerza, la secuencia; también se incluyen aspectos de orden cognitivo como la organización temporal, la atención, la memoria, el seguimiento de instrucciones, etc. La fuerza muscular necesaria para llevar a cabo este tipo de actividades está catalogada como baja y moderada, siendo ideal para trabajar con personas que manifiestan debilidad física marcada para que progresivamente la recuperen hasta donde sea posible hacerlo. Se puede mejorar la fuerza de las pinzas (pelando alimentos pequeños), la fuerza de prensión (cortando alimentos grandes), la pronosupinación (vertiendo líquidos), ejercitar la musculatura del hombro (amasando o extendiendo la masa de una pizza), entre otros.

Por otra parte, para analizar de forma sintética el papel de la terapia ocupacional en la mejora de AVD, De Conick y cols. (2017) Ilevaron a cabo un metaanálisis en el cual se buscaba verificar la eficacia de la T0 en esta población clínica.

Los autores seleccionaron nueve artículos en los cuales se evaluaba el efecto que tenían diversos programas de TO sobre los ancianos frágiles. La cantidad de participantes acumulados en los estudios abarcaba 3163 personas. Aquello que se pensaba medir en los estudios era la dificultad funcional en AVD, caídas o ambas. Dentro de los artículos incluidos existía una variación en la distribución, cantidad, naturaleza y duración de las sesiones de TO. Usualmente, las intervenciones consistían en varias sesiones de T0 donde el terapeuta acudía al hogar, seguidas de sesiones que buscaban fortalecer lo aprendido y concluían con un posterior seguimiento.

Las intervenciones consistieron principalmente en evaluación, educación e información, estrategias de prevención, ejercicios, uso de tecnología de asistencia, modificaciones de peligros del hogar, servicios, anuncios de servicios y sesiones de seguimiento.

En otro estudio liderado por el mismo autor e incluido en el análisis de De Conick y cols. (2017), Clemson y cols. (2012) trabajaron con una muestra de ancianos frágiles de 70 o más años que habían tenido una caída en los últimos 12 meses, poniendo a prueba un programa de entrenamiento para prevención de caídas. Dicha intervención fue llevada a cabo de manera conjunta por fisioterapeutas y terapeutas ocupacionales entrenados.

La intervención consistió principalmente en la puesta de un programa que los autores denominaron LiFE (Lifestyle integrated Functional Excercise), que consiste en realizar una integración de ejercicios en la vida diaria y no como un programa de ejercicios que debe ser llevado a cabo en una hora determinada como una rutina. Este programa supone principalmente el fortalecimiento del equilibrio y el aumento de la fuerza de las extremidades inferiores. Adicionalmente, la aplicación de este programa fue comparada con otros dos grupos: el primero era 


\section{TERAPIA OCUPACIONAL EN FRAGILIDAD: UNA APROXIMACIÓN BIBLIOGRÁFICA}

un grupo de ancianos que recibía un entrenamiento de ejercicios de otro estilo (ejercicios programados en una cantidad de tiempo determinada durante el día), y el otro era considerado como un grupo control, es decir, ancianos que no recibieron ningún tipo de tratamiento.

El programa LiFE incluye dentro de sus estrategias fundamentales el mejoramiento del equilibrio; para ello, se plantean ejercicios como: «reducir la base del soporte», «cambiar el peso del cuerpo de pie a pie», «pasar por encima de objetos» y «cambiar de dirección al caminar». Dentro de las técnicas que se incluyen en «reducir la base del soporte» se puede trabajar de modo que se les entrene para que al Ilevar a cabo labores de cocina, 0 cualquier otra actividad, lo hagan estando de pie, y que cuando puedan hacerlo con facilidad, intenten hacer las mismas actividades manteniendo todo el peso apoyado en una sola pierna.

Dentro de las estrategias que se incluyen para el aumento de la fuerza están (recuérdese que estos son ejercicios que deben ser llevados a cabo en la vida diaria y no bajo un programa rígido estructurado): flexionar las rodillas; de pie sobre los dedos de los pies; subir escaleras; de pie sobre los talones; ponerse de pie después de estar sentado; caminar de lado; y apretar los músculos:

Con frecuencia, los terapeutas ocupacionales deben implantar este tipo de programas en pacientes que poseen riesgo de caídas y, si además se trata de una población frágil, se debe tener especial cuidado con ellos. En los estudios de Close y cols. (1999) y de Davidson, Bond, Dawson, Steen y Kenny (2005), Ios terapeutas ocupacionales son los encargados de realizar la evaluación de las condiciones físicas así como de la dependencia/independencia de la persona mayor para así poder plantear una intervención

Consecuentemente, en otro de los artículos incluidos en el estudio de De Conick y cols. (2017), en la investigación Ilevada a cabo por Clark y cols. (2012) se evidenció que la intervención tuvo diversas vertientes, tales como:

- Desarrollo de planes para sobreponerse a obstáculos diarios.

- Participación en actividades selectivas.

- Evaluación del uso del tiempo y de patrones de gasto de energía.

- Conservación de energía.

- Revisión de peligros del hogar, prevención de caídas, preparación de emergencia.

- Actividad física y mental en la vida diaria.

- Realización de rutinas.

- Establecimiento y fortalecimiento de las relaciones sociales.

Tras haber realizado un análisis sistemático de todos los artículos incluidos en su metaanálisis, De Conick y cols. (2017) concluyen que, aunque las intervenciones variaron significativaente, en todos ellos se infiere que la TO ayuda a los adultos mayores frágiles a mejorar el funcionamiento en las AVD, en la participación social y la movilidad. Se observó igualmente que el miedo a las caídas también disminuyó en aproximaciones que incluían a la TO como un vértice de intervención. Adicionalmente, los autores aseguran que la T0 ayudó a empoderar a las personas a través de la ejecución de actividades significativas y que estas consecuentemente ejecutaban sus actividades diarias de manera efectiva.

Se considera efectivo que estas terapias no se desarrollen exclusivamente en los centros de atención pública 0 privada de los ancianos, sino que deben ser ejercidas por los profesionales en las viviendas de los adultos mayores ya que estos pasan la mayor parte de su tiempo allí, reduciendo así los costos de profesionales sociosanitarios, personas de cuidado, hospitalizaciones e institucionalizaciones de esta población.

\section{DISCUSIÓN}

La bibliografía revisada sobre la acción de la terapia ocupacional sobre adultos mayores frágiles arroja que el cuidado de estos profesionales resulta indispensable al momento de realizar un plan de tratamiento. Los terapeutas ocupacionales en conjunción con terapeutas físicos, médicos y otros profesionales deben coordinar sus acciones para desarrollar planes efectivos de trato al paciente. 
La literatura científica existente sobre el tema, si bien es escasa, demuestra la efectividad de la T0 en el trato de los adultos mayores en situación de fragilidad. Los conceptos y procedimientos de intervención que se identifican en la bibliografía se fundamentan principalmente en un enfoque centrado en el cliente. De forma mucho más específica, los terapeutas ocupacionales deben entrenar a los adultos mayores a desarrollar, recuperar 0 compensar habilidades que redunden en una ejecución más fluida de sus AVD, contribuyendo también a través de la evaluación y mejoramiento de las condiciones materiales del lugar donde residen y proveyendo productos tecnológicos asistenciales.

Se encuentra de esta forma, que la intervención del terapeuta ocupacional busca desarrollar estrategias físicas, cognitivas y materiales que le permitan al adulto mayor revertir su fragilidad y mantenerse en una tasa esperada de ejecución de sus actividades diarias.

Las investigaciones revisadas plantean principalmente planes de intervención general basados en modelos o estrategias que guían la acción de los terapeutas ocupacionales en el trato con el adulto mayor; sin embargo, no se hacen referencias específicas sobre el plan de trabajo exacto (técnicas, ejercicios particulares, etc.) que debe implementarse en la práctica. Al ser un campo relativamente nuevo de estudio es esperable este tipo de lagunas en el conocimiento, no obstante, la importancia del trato hacia estos pacientes supondrá que paulatinamente se lleven a cabo más investigaciones y se planteen esquemas más específicos de seguimiento.

Mención especial requiere el lugar de realización de las intervenciones; en la mayoría de los estudios se propone que estas se lleven a cabo en el propio domicilio de la persona, es decir, se propone trabajar en entornos naturales de forma que la actividad que se quiere mejorar se realice en el mismo lugar en el que se realizaría habitualmente.

En lo referente a la tipología de las intervenciones, se muestra que se realizan intervenciones basadas en criterios de modificación del entorno que, en sí mismos, lo hacen más facilitador, en la prescripción y entrenamiento en el uso de productos de apoyo y en el entrenamiento de la propia actividad que se quiere mejorar.

Las actividades Ilevadas a cabo en los tratamientos se aúnan en dos grandes grupos: las intervenciones que trabajan desde el entrenamiento de la propia actividad para conseguir una mejoría en la misma, y las intervenciones que trabajan otro tipo de actividad (véase, por ejemplo, jardinería) a través de la cual se refuerzan los componentes (físicos, cognitivos, etc.) de las actividades que se quiere mejorar.

En todas las intervenciones se obtiene una mejoría significativa de la independencia funcional de las personas participantes; además, es destacable que en el estudio en el que se ha medido, se observa una disminución de la mortalidad en los dos años posteriores; sería interesante poder evaluar esto en diferentes estudios. Además, en varios estudios se pone de manifiesto que disminuyen las hospitalizaciones y las institucionalizaciones y, consecuentemente, el gasto sociosanitario que este tipo de pacientes supone tanto de manera pública como privada.

Destaca también el hecho de no haber localizado estudios sobre el tratamiento de la fragilidad desde la TO en personas mayores institucionalizadas, en cuyo caso, el domicilio habitual debería ser considerado la propia institución. Resulta importante ya que la incidencia de fragilidad en este tipo de pacientes es muy significativa.

\section{CONCLUSIONES}

A través del prisma de la T0, Ios resultados obtenidos en la revisión bibliográfica sobre T0 y fragilidad ponen de manifiesto que, aunque muy escasa, existe investigación que avala los buenos resultados de las intervenciones desde la T0 en personas en situación de fragilidad, pero no se ha encontrado evidencia científica que haya estudiado y concluido cuáles son los factores que más frecuentemente se encuentran afectados y necesitan intervención desde la TO. Estos factores serían los denominados aspectos del dominio y del proceso de la TO y, por Io tanto, pueden y deben ser intervenidos desde esta disciplina. 


\section{TERAPIA OCUPACIONAL EN FRAGILIDAD: UNA APROXIMACIÓN BIBLIOGRÁFICA}

\section{REFERENCIAS BIBLIOGRÁFICAS}

Clark, F., Jackson, J., Carlson, M., Chon, C., Cherry, B., Jordan-Marsh, M., ... Azen, S. (2012). Effectiveness of a lifestyle intervention in promoting the well-being of independently living older people: results of que well elderly 2 randomised controlled trial. Journal of Epidemiology and Community Health, 66(9), 782-790.

Clemson, L., Singh, M., Bundy, A., Cumming, R., Monollaras, K., O'Loughlin, P. y Black, D. (2012). Integration of balance and strength training into daily life activity to reduce rate of falls in older people (the LiFE study): randomised parallel trial. British Medical Association, 2012(345), e4547. Doi: 10.1136/bmj.e4547.

Close, J., Ellis, M., Hooper, R., Glucsman, E., Jackson, S. y Swift, C. (1999). Prevention of falls in the elderly trial (PROFET) a randomised controlled trial. The Lancet, 353(9147), 93-97.

Davidson, J., Bond, J., Dawson, P., Steen, I. y Kenny, R. (2005). Patients with recurrent falls attending Accident \& Emergency benefit from multifactorial intervention —a randomised controlled trial. Age and Ageing, 34(2), 162-168.

De Conick, L., Bekkering, G., Bouckaert, L., Declercq, A., Graff, M. y Aertgeerts, B. (2017). Home and community-based occupational therapy improves functioning in frail older people: a systematic review. Journal of the American Gerietrics Society, 65(8), 1863-1869.

Fisher, A., Atler, K. y Potts, A. (2007). Effectiveness of occupational therapy with frail community living older adults. Scandinavian Journal of Occupational Therapy, 14(4), 240-249.

Fried, L., Borhani, N., Enright, P., Furberg, C., Gardin, J., Kronnal, R., ... Weiler, P. (1991). The cardiovascular health study: design end rationale. Annals of Epidemiology, 1(3), 163-276.

Fried, L. P., Tangen, C. M., Walston, J. D., Newman, A. B., Hirch, A., Gottdiener, J., ... McBurnie, M. A. (2001). Frailty in older adults: evidence for a phenotype. Journals of Gerontology. Series A: Biological Sciences and Medical Sciences, 56A(3), M146-M156.

Gitlin, L., Laraine, W., Dennis, M., Carcoran, M., Schinfeld, S. y Hauck, W. (2006). A randomized trial of a multicomponent home intervention to reduce functional difficulties in older adults. Journal of the American Geriatrics Society, 54(5), 809-816.

Ledesma, I. J. (2015). Sarcopenia y desempeño ocupacional de las actividades básicas de la vida diaria en mayores institucionalizados. Revista asturiana de Terapia Ocupacional, (12), 22-29.

Mann, W., Ottenbacher, K., Fraas, L., Tomita, M. y Granger, C. (1991). Effectiveness of assistive technology and environmental interventions in maintaining independence and reducing home care cost for the frail elderly. Archives of Family Medicine, 8(3), 210-117. 